\title{
Polylactic Acid Nonwoven Fabric Surface Modified with Stereocomplex Crystals for Recyclable Use in Oil/Water Separation
}

Chenxi Zhu ${ }^{1}$, Wei Jiang ${ }^{1 *}$, Jinglei Hu ${ }^{2 *}$, Ping Sun ${ }^{1}$, Aimin Li ${ }^{1}$, Quanxing Zhang ${ }^{1}$

1, State Key Laboratory of Pollution Control and Resources Reuse, School of the

Environment, Nanjing University, Nanjing 210023, China

2, Kuang Yaming Honors School \& Institute for Brain Sciences, Nanjing University, Nanjing 210023, China

*Corresponding author: jiangwei@nju.edu.cn;

hujinglei@nju.edu.cn; 


\section{Preparation of the modified PLA nonwoven fabric with different concentrations}

of PDLA solution

First, a certain amount of PDLA particles were mixed with dichloromethane to prepare a $0.05 \mathrm{wt} \%$ PDLA solution called solution A. Absolute ethyl alcohol, n-butyl alcohol, and nbutyl acetate (volume ratio of 1:1:1) were mixed and stirred at $500 \mathrm{rpm}$ for 30 minutes to prepare the nonsolvent called solution B. Second, 2.75, 2.5, 2.25, 2, 1.75 and 1.5 times the volume of solution B was slowly added to solution A and stirred for 30 minutes. Finally, 15 $\mathrm{ml}$ of the above solution was pipetted into a Petri dish containing the PLLA nonwoven fabric and then dried at room temperature for $48 \mathrm{~h}$. These samples were named PDLA-1, PDLA-2, PDLA-3, PDLA-4, PDLA-5, PDLA-6, and the unmodified PLLA nonwoven fabric was called PDLA-0.

\section{Preparation of the modified PLA nonwoven fabric and modified PP nonwoven}

\section{fabric in different solutions}

First, a certain amount of PDLA particles and PLLA particles were mixed separately with dichloromethane to prepare a $0.05 \mathrm{wt} \%$ PDLA solution and a $0.05 \mathrm{wt} \%$ PLLA solution. Absolute ethyl alcohol, n-butyl alcohol, and n-butyl acetate (volume ratio of 1:1:1) were mixed and stirred at $500 \mathrm{rpm}$ for 30 minutes to prepare the nonsolvent called solution A. Subsequently, 1.5 times the volume of solution A was added into the above PLLA solution and PDLA solution and stirred for 30 minutes to prepare solution B and solution C. Solutions A and B, and C were added into Petri dishes containing the PLLA nonwoven fabric; at the same time, $15 \mathrm{ml}$ of solution B was added into the Petri dish containing the PP nonwoven fabric. These samples were dried at room temperature for $48 \mathrm{~h}$ and named as PLLA nonwoven fabric modified with nonsolvent, PLLA nonwoven fabric modified with PDLA, PLLA nonwoven fabric modified with PLLA, and PP nonwoven fabric modified with PDLA. 


\section{Preparation of the modified PLA nonwoven fabric with PLLA nonwoven fabric}

\section{of different densities}

First, a certain amount of PDLA particles were mixed with dichloromethane to prepare a $0.05 \mathrm{wt} \%$ PDLA solution called solution A. Absolute ethyl alcohol, n-butyl alcohol, and nbutyl acetate (volume ratio of 1:1:1) were mixed and stirred at $500 \mathrm{rpm}$ for 30 minutes to prepare the nonsolvent called solution B. Second, 1.5 times the volume of solution B was slowly added into solution A and stirred for 30 minutes. Finally, $15 \mathrm{ml}$ of the above solution was pipetted into Petri dishes containing PLLA nonwoven fabric with a density of $80 \mathrm{~g} / \mathrm{m}^{2}$ and $100 \mathrm{~g} / \mathrm{m}^{2}$ and then dried at room temperature for $48 \mathrm{~h}$. These samples were named as 80 $\mathrm{g} / \mathrm{m}^{2}$ PLA and $100 \mathrm{~g} / \mathrm{m}^{2}$ PLA, respectively.

Preparation of the modified PLA nonwoven fabric with different concentrations of PLLA solution

First, a certain amount of PLLA particles were mixed with dichloromethane to prepare a $0.05 \mathrm{wt} \%$ PDLA solution called solution A. Absolute ethyl alcohol, n-butyl alcohol, and nbutyl acetate (volume ratio of 1:1:1) were mixed and stirred at $500 \mathrm{rpm}$ for 30 minutes to prepare the nonsolvent called solution B. Second, 1.5, 2 and 2.5 times the volume of solution B was slowly added to solution A and stirred for 30 minutes. Finally, $15 \mathrm{ml}$ of the above solution was pipetted into a Petri dish containing the PLLA nonwoven fabric and then dried at room temperature for $48 \mathrm{~h}$. These samples were named PLLA-1, PLLA-2, PLLA-3. 


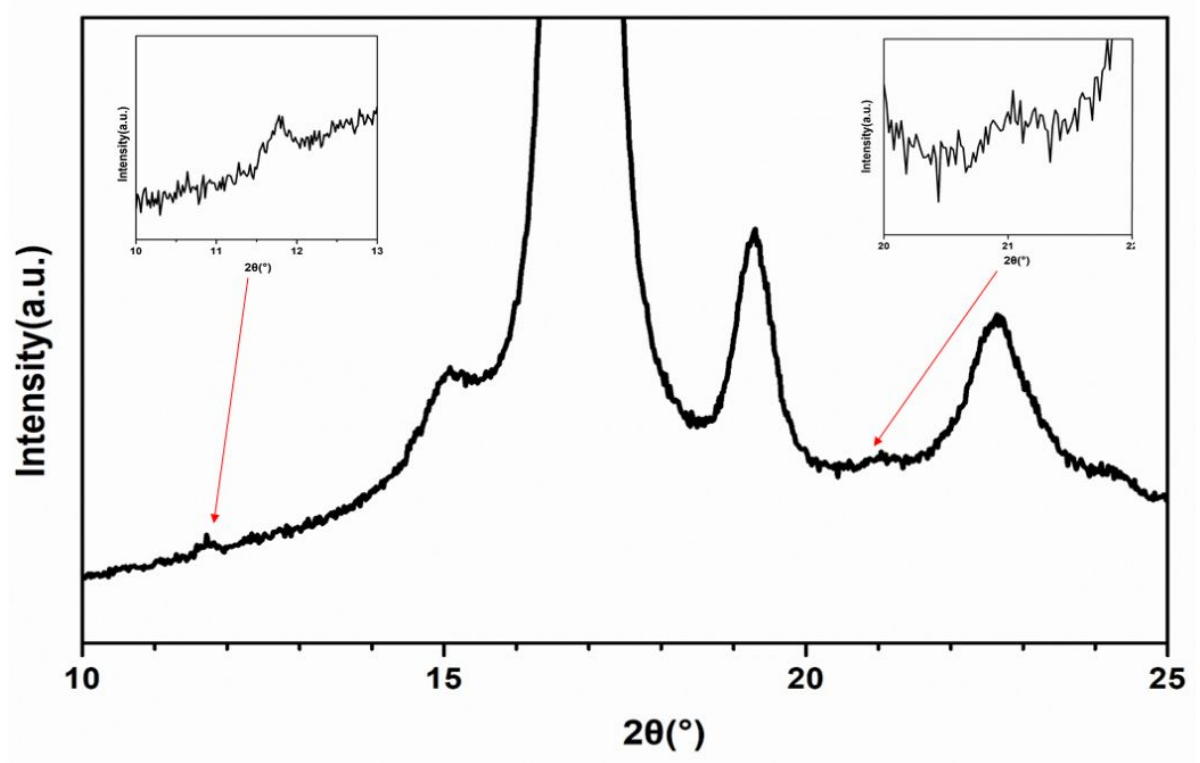

Figure S1. XRD patterns of the modified PLLA nonwoven fabric. Inset: Enlarged 10$13^{\circ}$ and $20-22^{\circ}$ XRD heating curves of modified nonwoven fabric. 

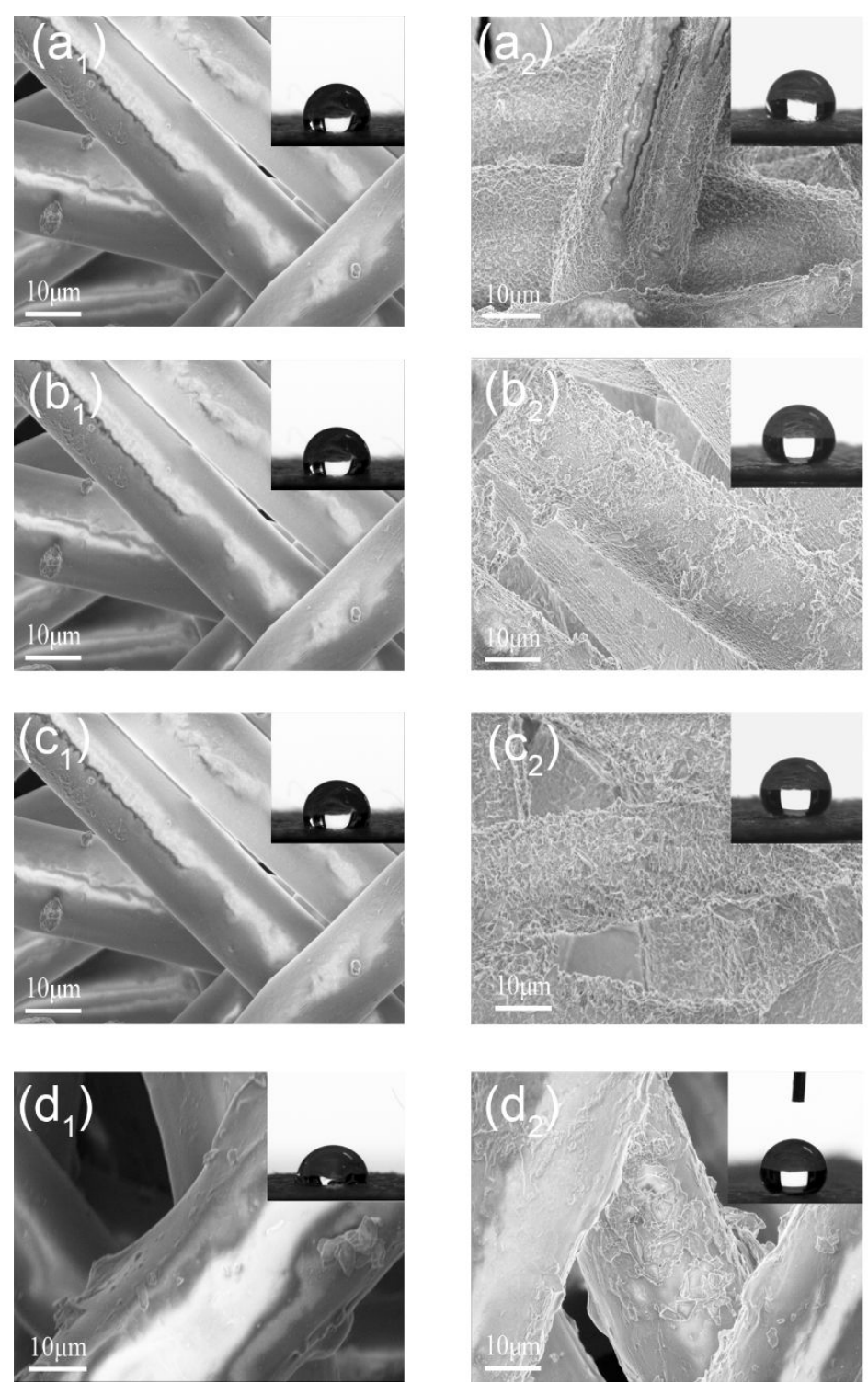
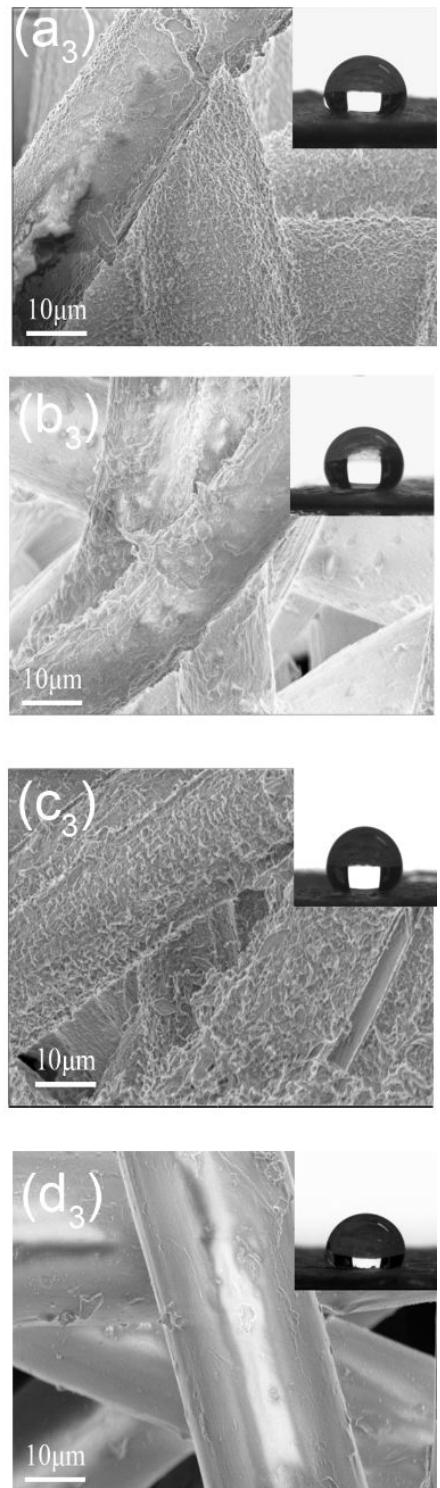

Figure S2 ESEM images and static WCA measurements of raw PLA nonwoven fabric (a1,b1,c1),

PLA nonwoven fabric modified with nonsolvent (a2), PLA nonwoven fabric modified with nonsolvent after ultrasonication (a3), PLA nonwoven fabric modified with PLLA (b2), PLA nonwoven fabric modified with PLLA after ultrasonication (b3), PLA nonwoven fabric modified with PDLA (c2), PLA nonwoven fabric modified with PDLA after ultrasonication (c3), raw PP nonwoven fabric(d1), PP nonwoven fabric modified with PDLA (d2) and PP nonwoven fabric 
modified with PDLA after ultrasonication (d3) 
In practical applications, the physical stability of the nonwoven fabric is an important factor limiting the ability of oil/water separation. In this experiment, we used 150 mesh sandpaper to measure the abrasion resistance of the modified nonwoven fabric, and the measuring device was designed in our lab. As shown in Figure S3a, the modified nonwoven fabric was placed under a 200 $\mathrm{g}$ iron block. The applied force on the iron block was $5 \mathrm{~N}$, and the frication distance was $20 \mathrm{~cm}$. Twenty cycles of abrasion on the nonwoven fabric were performed. The WCA was measured after every fifth cycles . As shown in Figure S3c, the changes in the WCA values are not significant, and the contact angle of the nonwoven fabric remained as high as $146.0 \pm 1.1^{\circ}$ after 20 cycles. Figures $\mathrm{S} 3 \mathrm{~b}$ and S3d describe the surface of the nonwoven fabric after the abrasion test.
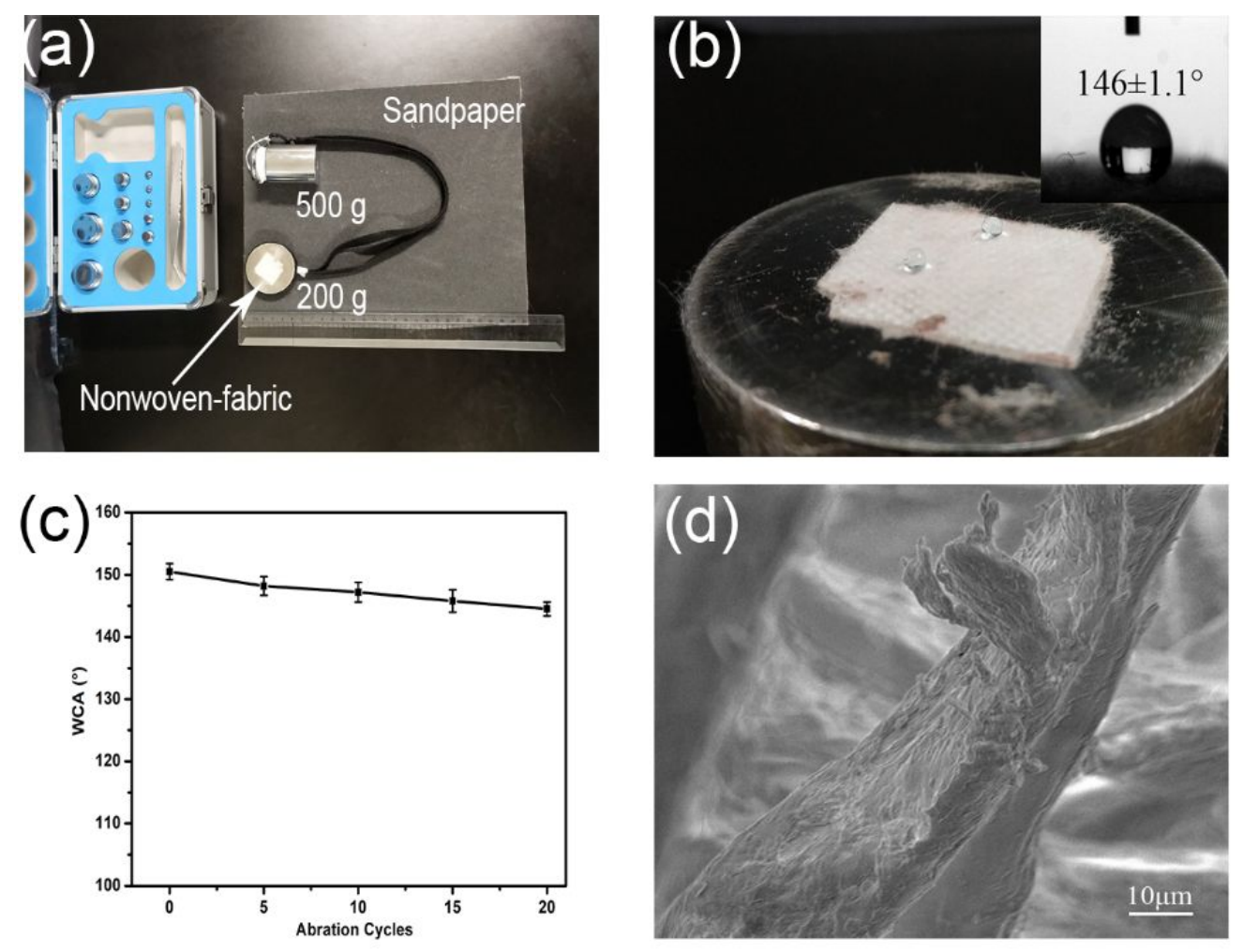

Figure S3. Abrasion resistance of the modified PLA nonwoven fabric, (a) photograph 
of the homemade abrasion test device, (b) photograph of the membrane after the abrasion test. (c)The change of WCA with the increase of friction times. (d)ESEM images of the modified PLA nonwoven fabric after the abrasion test. 


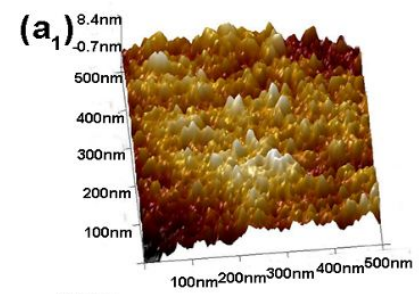

$\left(b_{1}\right)_{1.3}^{22.7}$

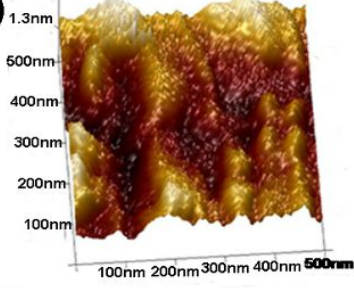

$\left(c_{1}\right)^{31}$

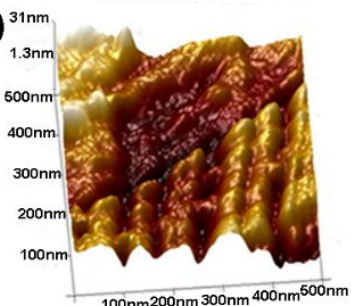

$\left(d_{1}\right)^{41.6 \mathrm{~nm}}$

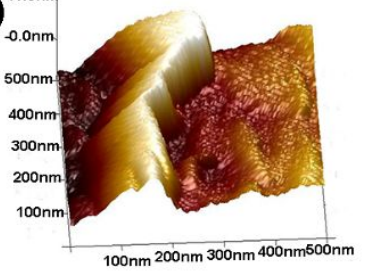

$\left(e_{1}\right)^{6}$
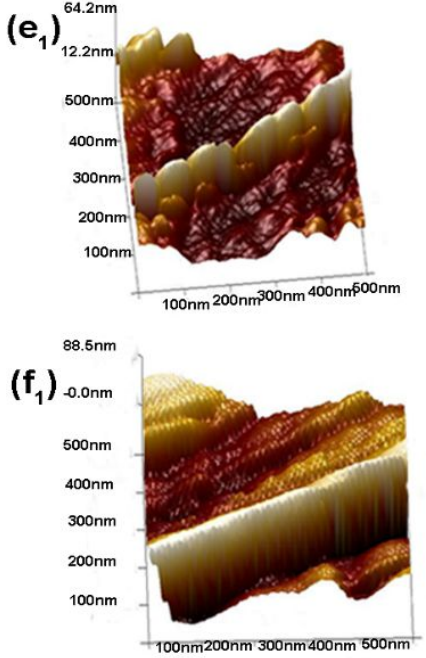

$\left(g_{1}\right)_{2.9 n m}^{93.6 \mathrm{~nm}}$

$500 \mathrm{~nm}$

$400 \mathrm{~nm}$

$300 \mathrm{~nm}$

$200 \mathrm{~nm}$

$100 \mathrm{~nm}$

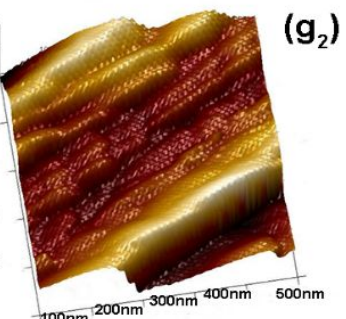

$\left(a_{2}\right)$

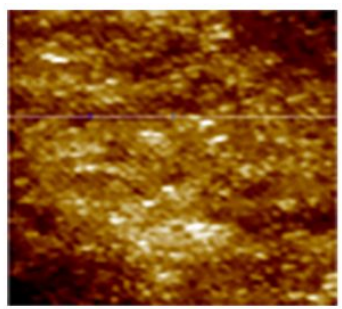

$\left(b_{2}\right)$

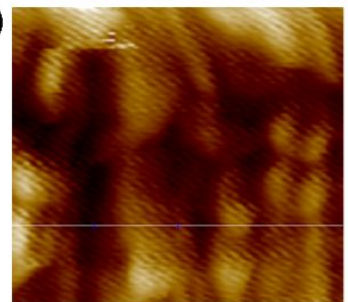

$\left(c_{2}\right)$

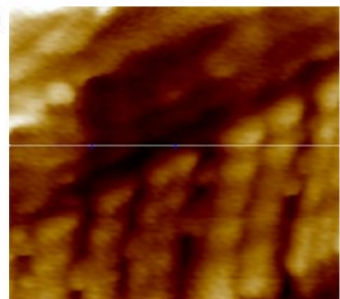

$\left(d_{2}\right)$

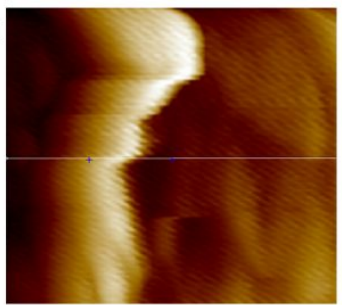

$\left(e_{2}\right)$

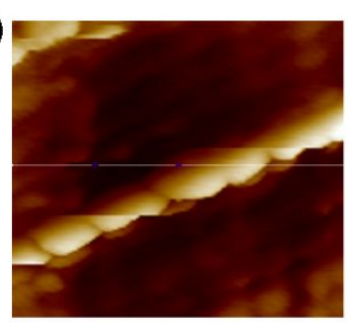

$\left(f_{2}\right)$

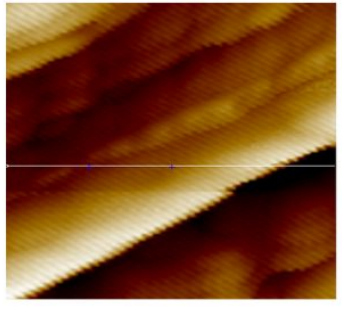

$\left(g_{2}\right)$

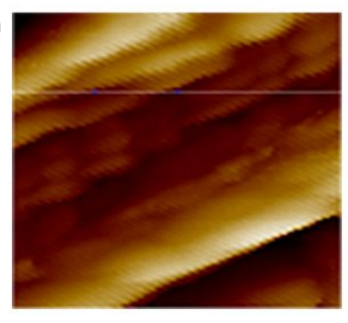

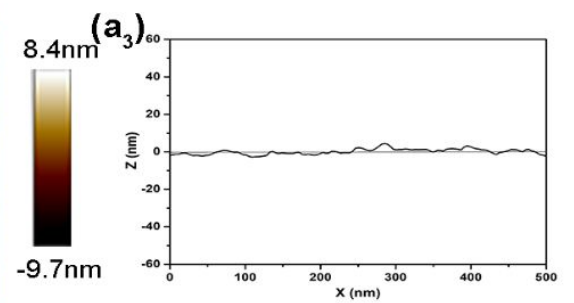
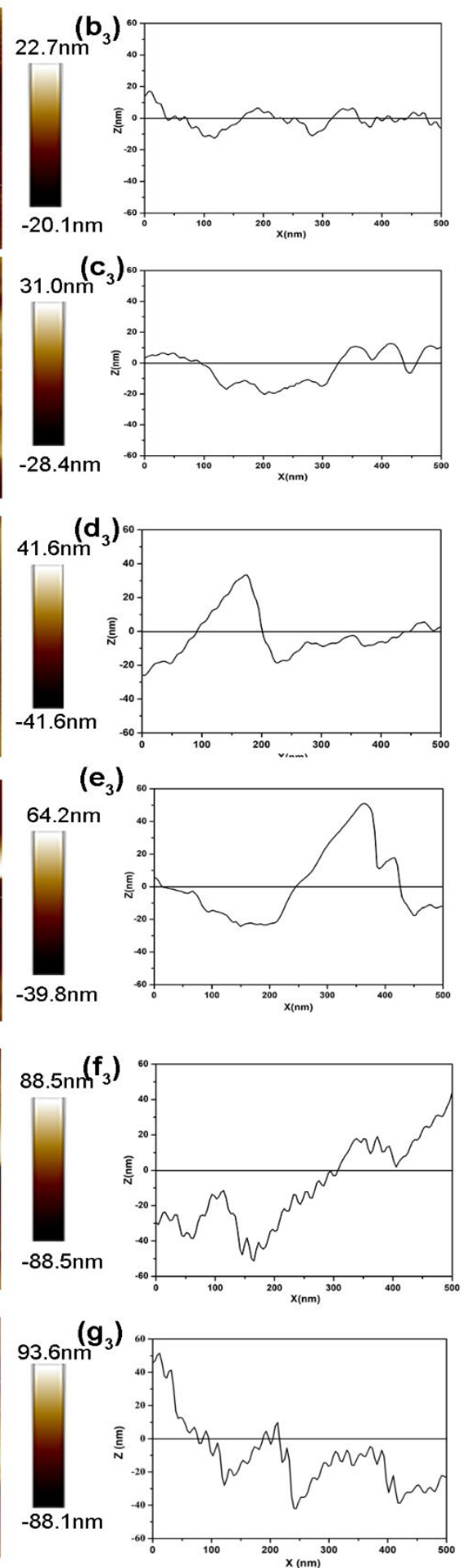

Figure S4. $\left(a_{1}\right)$ Three-dimensional (3-D) AFM images, $\left(a_{2}\right)$ two-dimensional (2-D) AFM images 
and $\left(\mathrm{a}_{3}\right)$ section roughness of PDLA- $0 .\left(\mathrm{b}_{1}\right)$ Three-dimensional (3-D) AFM images, $\left(\mathrm{b}_{2}\right)$ twodimensional (2-D) AFM images and $\left(\mathrm{b}_{3}\right)$ section roughness of PDLA-1.( $\left.\mathrm{c}_{1}\right)$ Three-dimensional (3D) AFM images, $\left(c_{2}\right)$ two-dimensional (2-D) AFM images and $\left(c_{3}\right)$ section roughness of PDLA-2. $\left(\mathrm{d}_{1}\right)$ Three-dimensional (3-D) AFM images, $\left(\mathrm{d}_{2}\right)$ two-dimensional (2-D) AFM images and $\left(\mathrm{d}_{3}\right)$ section roughness of PDLA-3.( $\left.\mathrm{e}_{1}\right)$ Three-dimensional (3-D) AFM images, $\left(\mathrm{e}_{2}\right)$ two-dimensional (2D) AFM images and $\left(e_{3}\right)$ section roughness of PDLA-4. $\left(f_{1}\right)$ Three-dimensional (3-D) AFM images, $\left(\mathrm{f}_{2}\right)$ two-dimensional (2-D) AFM images and $\left(\mathrm{f}_{3}\right)$ section roughness of PDLA-5. $\left(\mathrm{g}_{1}\right)$ Threedimensional (3-D) AFM images, $\left(\mathrm{g}_{2}\right)$ two-dimensional (2-D) AFM images and $\left(\mathrm{g}_{3}\right)$ section roughness of PDLA-6. 
Table S1. Measurement results of water contact angle(WCA) and roughness of different samples

\begin{tabular}{cccc}
\hline & WCA & \multicolumn{2}{c}{ roughness } \\
\cline { 3 - 4 } samples & $\left(^{\circ}\right)$ & $\begin{array}{c}\text { average surface } \\
\text { roughness(Ra) } \\
(\mathrm{nm})\end{array}$ & $\begin{array}{c}\text { root-mean-square } \\
\text { roughness(Rq) } \\
(\mathrm{nm})\end{array}$ \\
\hline PDLA-0 & $125.2 \pm 1 . .3$ & $3.04 \pm 1.48$ & $3.73 \pm 1.67$ \\
PDLA-1 & $135.4 \pm 1.6$ & $8.10 \pm 4.15$ & $9.90 \pm 4.88$ \\
PDLA-2 & $138.3 \pm 1.7$ & $8.10 \pm 2.45$ & $10.70 \pm 3.45$ \\
PDLA-3 & $140.4 \pm 2$ & $7.40 \pm 4.35$ & $9.49 \pm 5.88$ \\
PDLA-4 & $143.2 \pm 1.9$ & $15.14 \pm 7.81$ & $18.11 \pm 9.31$ \\
PDLA-5 & $148.2 \pm 1.4$ & $38.04 \pm 16.81$ & $47.48 \pm 19.91$ \\
PDLA-6 & $150.1 \pm 1.8$ & $44.42 \pm 20.73$ & $55.49 \pm 22.95$ \\
\hline
\end{tabular}


The oil/water separation performance is closely related to the surface superhydrophobicity. The surface wettability of PLA nonwoven fabrics was characterized by an optical contact angle meter. As shown in Figure S5a, the WCA of raw PLA nonwoven fabric is $120 \pm 1.8^{\circ}$ with no significant hydrophobicity, which is not suitable for use in the separation of oil/water mixtures. After modification, the WCA value of the modified PLA nonwoven fabric was $150.5 \pm 1.3^{\circ}$. Under these conditions, water droplets cannot easily penetrate into the nonwoven fabric. Interestingly, the oil contact angle of the both raw and modified PLLA nonwoven fabric were $0^{\circ}$ as shown in Figure S5b, which may be related to the natural property of the polylactic acid and the aperture of the PLA nonwoven fabric. As shown in Figure S5c, a water droplet formed a sphere with a WCA value of $150.5^{\circ}$. After standing in the air for thirty minutes, the droplet maintained its spherical shape, which indicated that the modified nonwoven fabric possessed remarkable superhydrophobic stability. The final water penetration time exceeded forty minutes. By changing the volume ratio of the PDLA solution and the nonsolvent, the static water penetration time of the modified PLA nonwoven fabric changed accordingly. As the volume ratio decreased, the static water penetration time correspondingly decreased. It is possible that as the amount of poor solvent increased, the content of PDLA in the obtained solution decreased, and agglomeration occurred in the rich phase. The generation of the stereocomplex crystals was reduced, and they were distributed unevenly. Furthermore, the modified nonwoven fabric remained superhydrophobic after ten cycles, which shows the durability of the wetting behaviors (Figure S4d). 

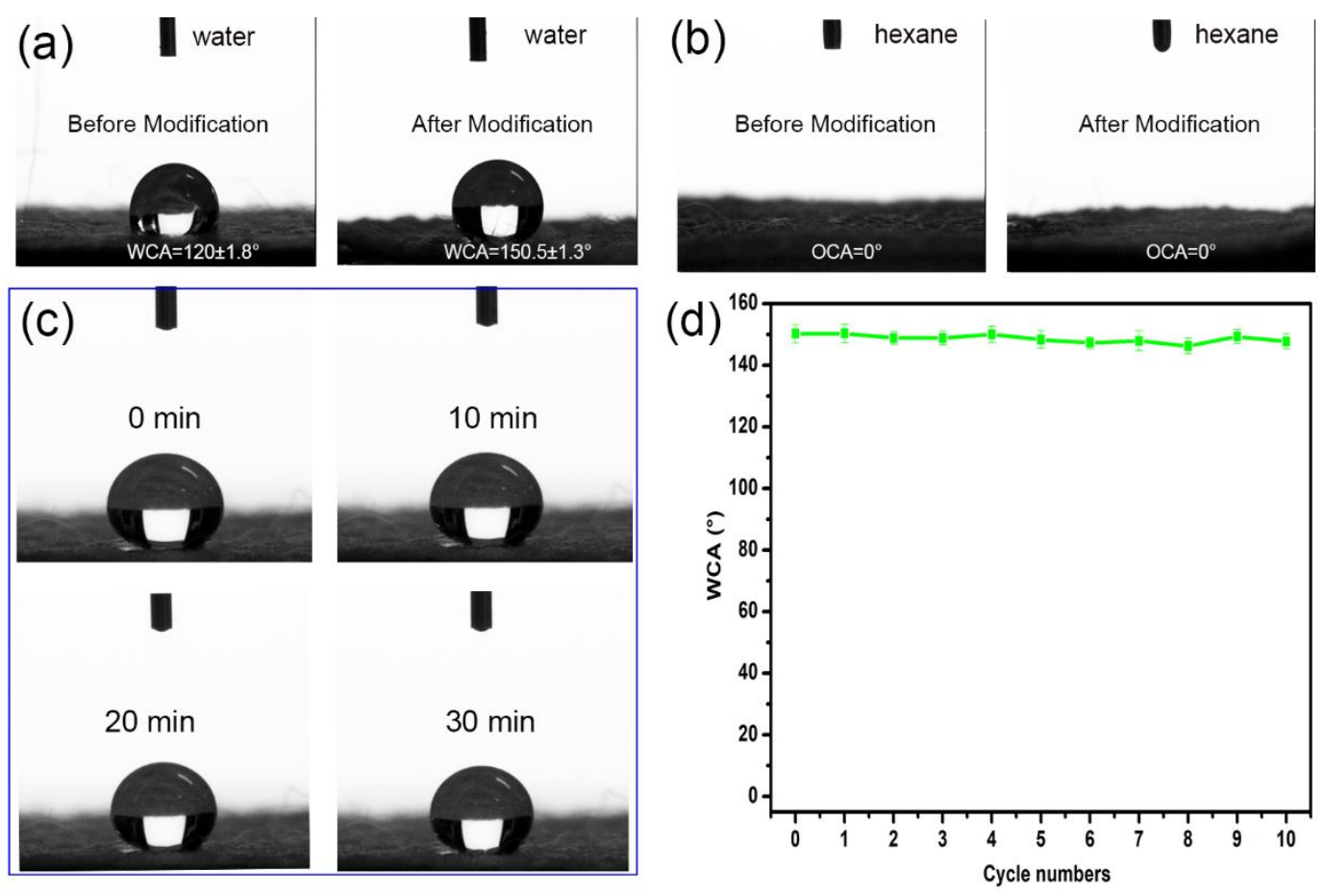

Figure S5 (a)Static water contact angle(WCA) measurements for raw PLA nonwoven fabric and modified PLA nonwoven fabric.(b) Static oil contact angle(OCA) measurements for raw PLA nonwoven fabric and modified PLA nonwoven fabric (c)Still images of WCA measurement of a water droplet on the modified PLA nonwoven fabric for 30 minutes. (d)The changes in WCA during cycle use of the modified nonwoven fabric. 


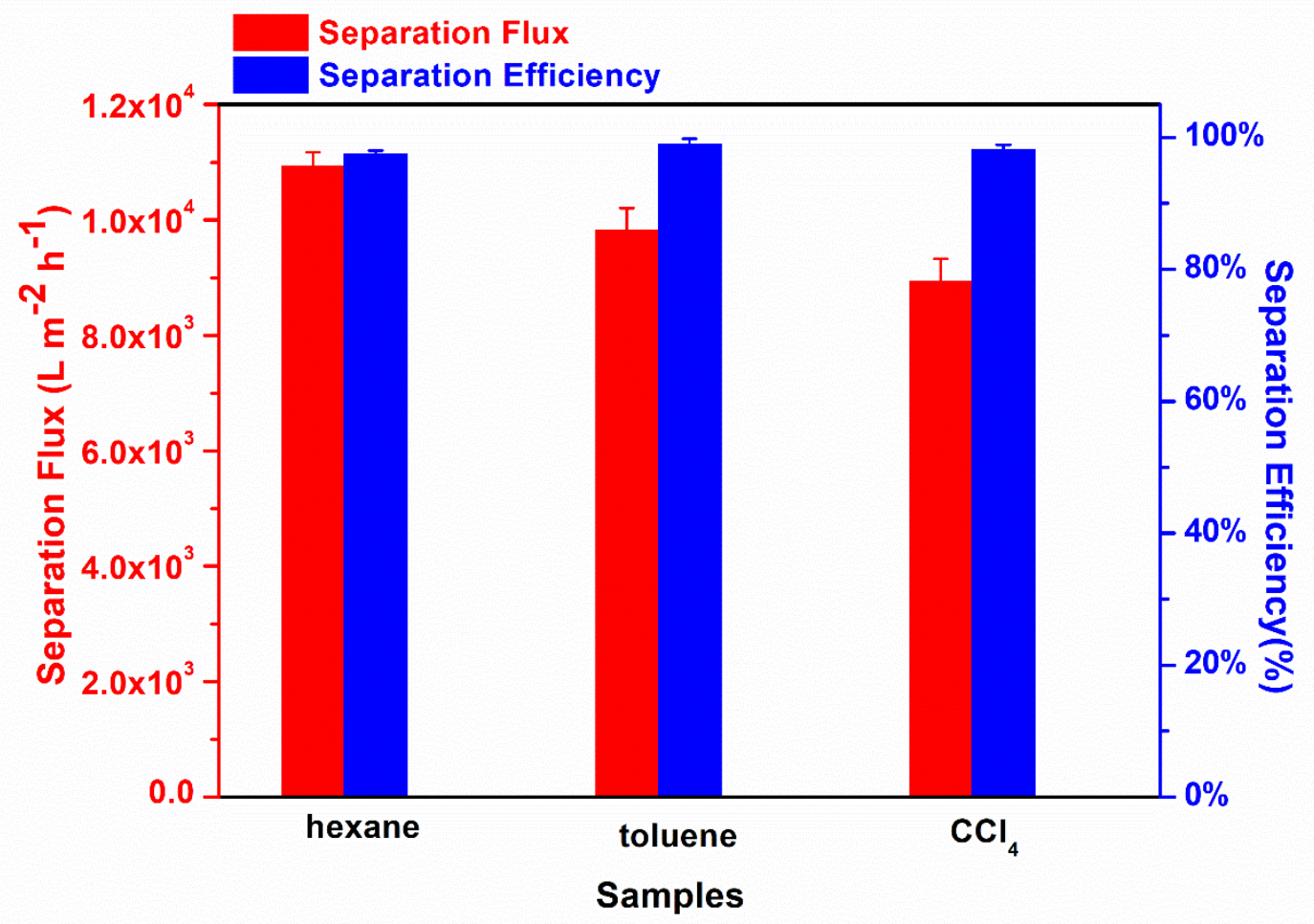

Figure S6 The separation fluxes and separation efficiency of organic solvents /water mixture passing through the modified PLLA nonwoven fabric. 

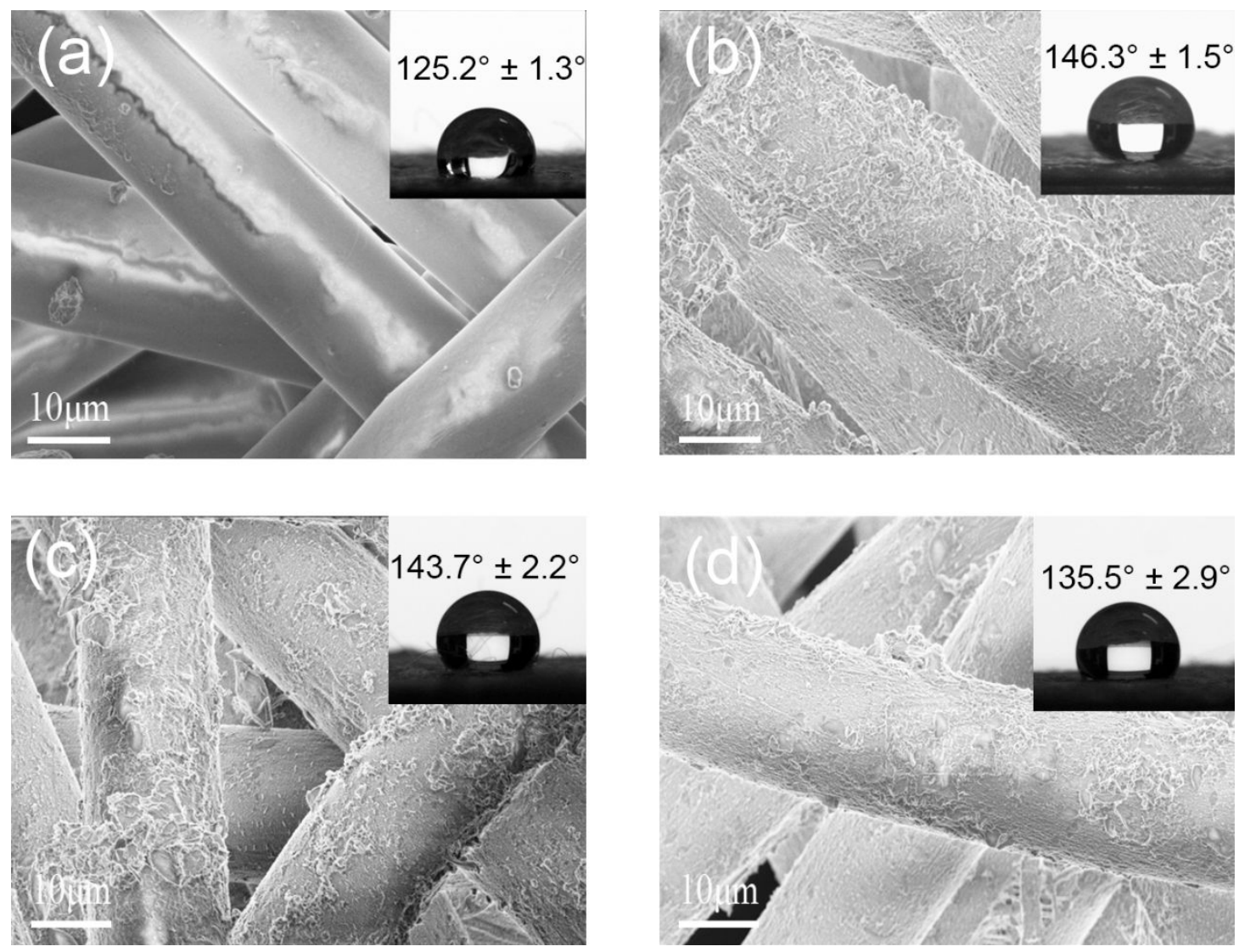

Figure S7 ESEM images and static WCA measurements of raw PLA nonwoven fabric(a), PLLA1(b),PLLA-2(c) and PLLA-3(d). 

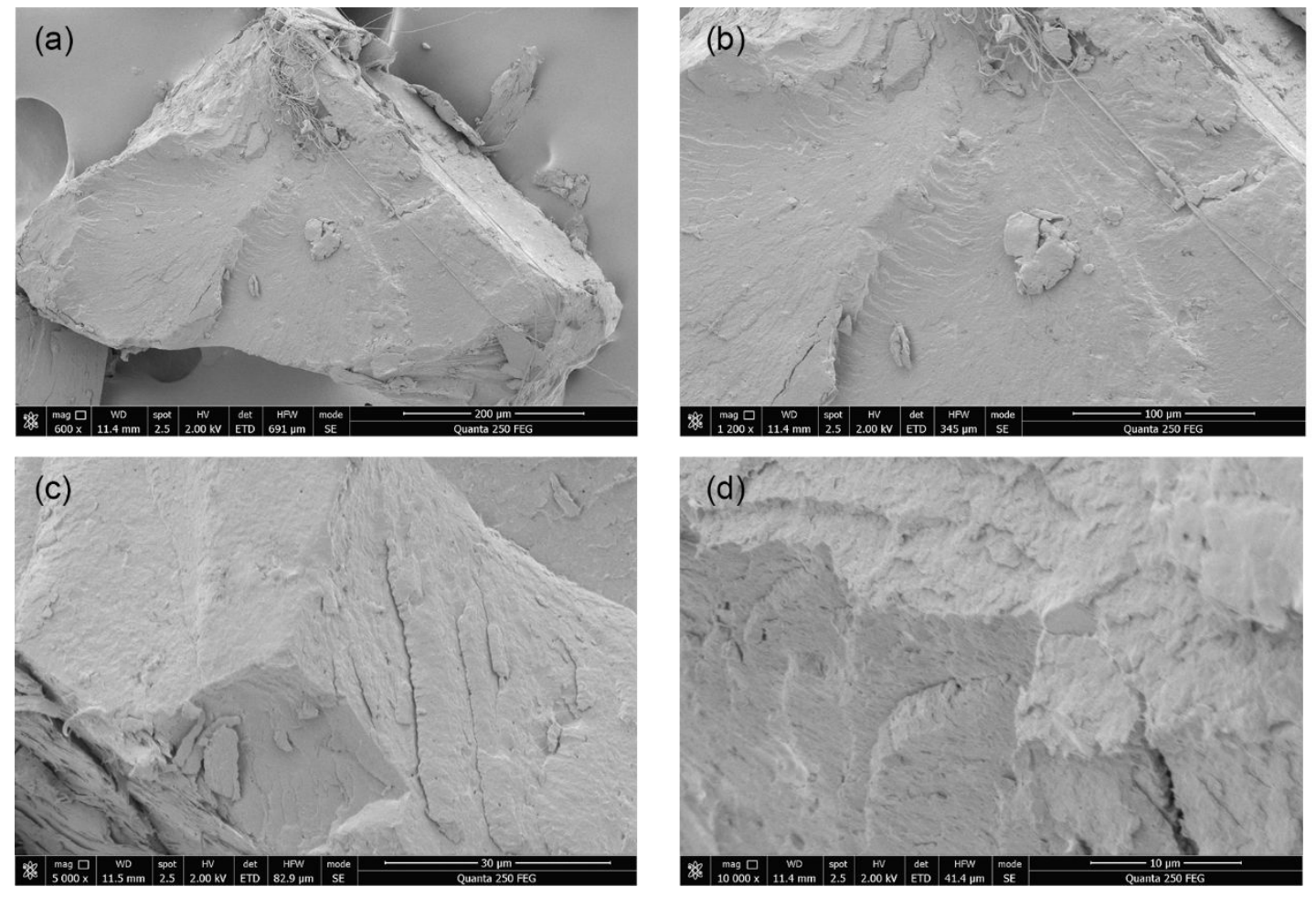

Figure S8 ESEM images with different magnification of PDLA nanoparticle (a)600×, (b), 1200

$\times(c) 5000 \times(d) 10000 \times$. 